\title{
Analytical Calculation of the Magnetic Field Created by Permanent-Magnet Rings
}

\author{
R. Ravaud, G. Lemarquand, V. Lemarquand, and C. Depollier \\ Laboratoire d'Acoustique de l'Universite du Maine UMR CNRS 6613, 72085 Le Mans Cedex 9, France
}

\begin{abstract}
We present analytical formulations, based on a coulombian approach, of the magnetic field created by permanent-magnet rings. For axially magnetized magnets, we establish the expressions for the three components. We also give the analytical 3-D formulation of the created magnetic field for radially magnetized rings. We compare the results determined by a 2-D analytical approximation to those for the 3-D analytical formulation, in order to determine the range of validity of the 2-D approximation.
\end{abstract}

Index Terms-Analytical calculation, axial magnetization, magnetic field, permanent-magnet rings, radial magnetization.

\section{INTRODUCTION}

$\mathbf{P}$ ERMANENT magnets are used nowadays in many applications, and the general need for dimensioning and optimizing leads to the development of calculation methods, whose first step is often to calculate the magnetic field created by the magnets. Two major kinds of applications can be identified: the ones which use parallelepipedic magnets and the ones which use cylindrical magnets. Parallelepipedic magnets are easy to produce and to magnetize, and the magnetic field they create is also more easily calculated. Geometrical methods have been proposed by Leupold [1] to calculate high uniform magnetic field sources [2] using wedge-shaped magnets-with sections presenting wedges, or angles lower than $90^{\circ}$-and extended from the Halbach "magical structure" [3]. Analytical approaches are of primary importance for the design of many devices, and the need for analytical methodologies is emphasized in all applications, from the generation of remote fields for MRI [4] or field gradients [5], to the microactuators [6] and diamagnetic levitation devices [7], not forgetting all the electrical motors topologies [8] and the sensors for mechanical data such as position or torque [9], [10].

Analytical approaches were proposed by Marinescu [11] and analytical 2-D and 3-D solutions were given by Yonnet [12] and by Bancel [13] for the field created by parallelepipedic magnets. These calculations were applied to the study of devices such as loudspeaker motors with no leakage [14], [15], guitar pickups [16], magnetic couplings, and bearings [17]. It is noticeable that in some applications, such as permanent-magnet motors [18] or magnetic couplings [19], tiles or ring magnets were replaced by parallelepipedic magnets in order to be able to calculate and optimize the structures as the required cylindrical formulations were not established yet.

But in devices of small size [20], radially magnetized ring magnets have to be used and hence such structures have to be studied. The case of a magnet ring axially magnetized was analytically studied by Durand [21]. More recently, Furlani [22] presented a semianalytical calculation and an analytical calculation of the field in the air gap of tubular permanent-magnet machines was presented by Zhilichev [23].

Digital Object Identifier 10.1109/TMAG.2008.923096
The case of radially magnetized sectors or rings, which corresponds to many permanent-magnet motor topologies, has already been considered. Furlani [24] proposed a semianalytical formulation based on the amperian model of the magnets which needs two numerical integrations. Rakotoarison [25] proposes a semianalytical solution to calculate the whole magnetic field created by radially magnetized sectors by a Coulombian approach which reduces the numerical integration number to a single one. Selvaggi [26], [27] uses a multipole representation of the source to calculate analytically the external 3-D magnetic field with Green's functions. Azzerboni [28]-[33] uses the Legendre's functions to determine the magnetic field created by magnet rings and Babic [34]-[36] uses both Legendre's polynoms and Heumann functions to calculate the magnetic field components at any point in space (either singular or regular). This paper proposes a fully exact analytical 3-D formulation of the magnetic field created by magnet rings without using serial functions. First, this paper presents the analytical calculation of the magnetic field created by an axially magnetized ring. The approach uses a coulombian model of the magnet. Then, the paper proposes a 3-D analytical formulation of the field created by a radially magnetized ring. The magnet is modeled by a surface density of fictitious charges on the inner face of the ring. The effect of the outer face does not appear to simplify the expressions, but can be taken into account because the principle of linear superposition applies.

We consider the magnetic field created by only one face, and do not take the volumic charge density into account, contrary to Rakotoarison [25] who proposes a fast 3-D semianalytical expression of the field produced by an arc-shaped magnet. An exact 3-D analytical formulation of the solution is a priori attractive for optimization purposes. But as 3-D formulations are also far more complicated than 2-D formulations, the comparison of the results given by both formulations is interesting, in order to determine, for example, the range of validity of the 2-D analytical approximation and to establish when the 2-D approximation is sufficient and when the exact 3-D formulation is required. This paper shows that the 2-D approach cannot give acceptable results for the calculation of the far field of the magnet, whatever the curvature of the magnet is, and especially for the field at the center of the magnet ring, contrary to the 3-D approach. This result is particularly significant for some applications, where the field in the center of the ring is the important result. 


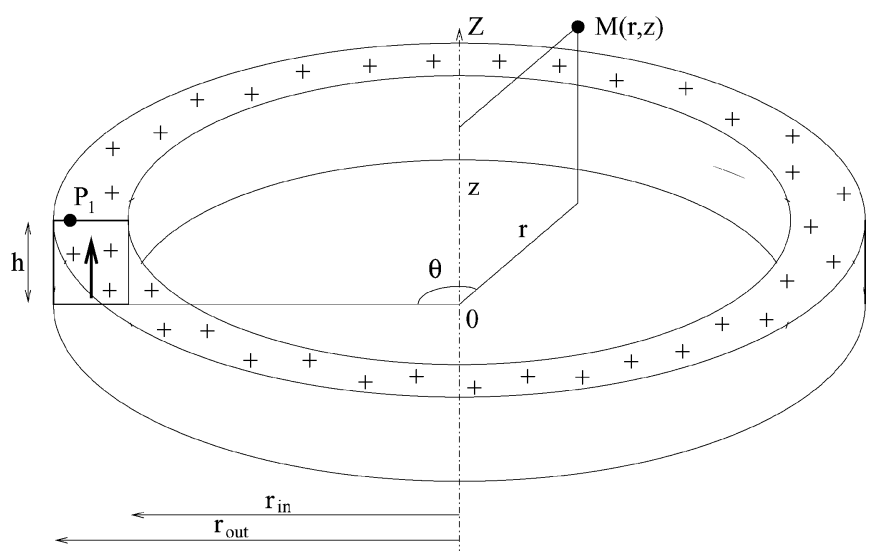

Fig. 1. Used geometry: it is a ring whose $z$ is an axis of symmetry, its inner radius is $r_{\text {in }}$, its outer radius is $r_{\text {out }}$, its height is $h$; its magnetic polarization is axial.

\section{Analytical Calculation of the Magnetic FIELD CREATED BY AXIALLY MAGNETIZED PERMANENT-MAGNET RINGS}

This section presents the analytical calculation of the magnetic field created by a permanent-magnet ring whose magnetic polarization is axial. The extensive calculation is presented only for one annular charged plane.

\section{A. Notation and Geometry}

The geometry considered and the related parameters appear in Fig. 1. The ring inner radius is $r_{\text {in }}$, the ring outer radius is $r_{\text {out }}$, and its height is $h$. The axis $z$ is an axis of symmetry. Calculations are obtained by the use of the Coulombian model. Consequently, the permanent-magnet ring is represented by two annular planes which correspond to the upper $(z=h)$ and lower $(\mathrm{z}=0)$ faces of the ring. The upper one is charged with a surface magnetic pole density $+\boldsymbol{\sigma}^{*}$; the lower one is charged with the opposite surface magnetic pole density $-\sigma^{*}$. All the illustrative calculations are done with $\sigma^{*}=\vec{J} \cdot \vec{n}=1 T$.

We only consider the upper face of the permanent-magnet ring to simplify the expression of the magnetic field. The total magnetic field created by the permanent-magnet ring is calculated by the application of the linear superposition principle. Let us consider a point $P_{1}$ on the ring upper face. The magnetic field $\vec{H}$ created by the ring upper face at any observation point $M(r, z)$ of the space is given by (1):

$$
\begin{aligned}
& \vec{H}(r, z)=\frac{\sigma^{*}}{4 \pi \mu_{0}} \int_{\theta=0}^{\theta=2 \pi} \int_{r_{1}=r_{\text {in }}}^{r_{1}=r_{\text {out }}} \frac{\overrightarrow{P_{1} M}}{\left|\overrightarrow{P_{1} M}\right|^{3}} r_{1} d r_{1} d \theta \\
& \vec{H}(r, z)=\frac{\sigma^{*}}{4 \pi \mu_{0}} \int_{\theta=0}^{\theta=2 \pi} \int_{r_{1}=r_{\text {in }}}^{r_{1}=r_{\text {out }}} \\
& \times \frac{\left(r-r_{1} \cos (\theta)\right) \vec{u}_{r}-r_{1} \sin (\theta) \vec{u}_{\theta}+\left(z-z_{1}\right) \vec{u}_{z}}{\left.\left(r^{2}+r_{1}^{2}-2 r r_{1} \cos (\theta)+\left(z-z_{1}\right)^{2}\right)\right)^{\frac{3}{2}}} r_{1} d r_{1} d \theta .
\end{aligned}
$$

\section{B. Components Along the Three Directions $\vec{u}_{r}, \vec{u}_{\theta}, \vec{u}_{z}$}

The integration of (1) leads to the magnetic field components along the three defined axes: $H_{r}^{(3 \mathrm{D})}(r, z), H_{\theta}^{(3 \mathrm{D})}(r, z)$,

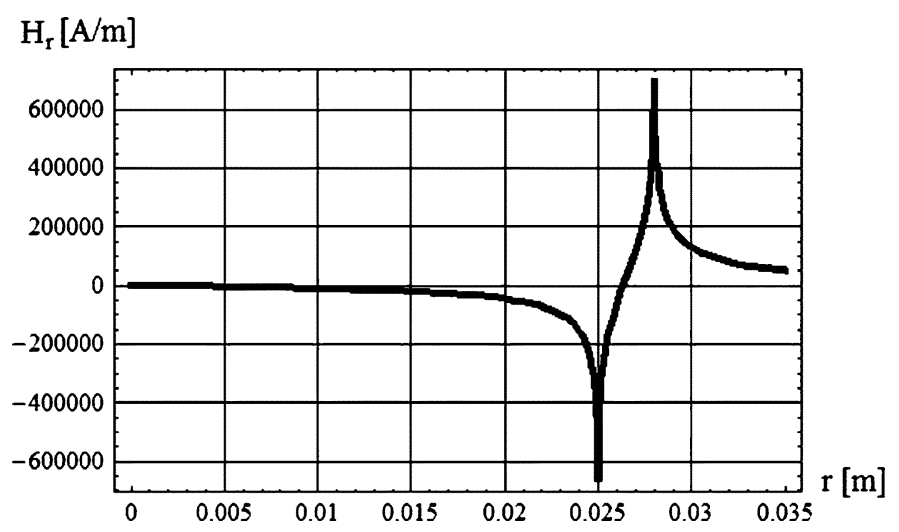

Fig. 2. The radial component $H_{r}^{\text {(durand) }}(r, z)$ is a function of the radial distance $r$; the observation height is $z=3 \mathrm{~mm} ; r_{\text {in }}=25 \mathrm{~mm}, r_{\text {out }}=28 \mathrm{~mm}$.

and $H_{z}^{(3 \mathrm{D})}(r, z)$ (expressed in $\left.\mathrm{A} / \mathrm{m}\right)$ which are given by the expressions (3), (4), and (9).

\section{Azimuthal Component $H_{\theta}^{(3 \mathrm{D})}(r, z)$}

The azimuthal component $H_{\theta}^{(3 \mathrm{D})}(r, z)$ equals 0 on account of the cylindrical symmetry

$$
H_{\theta}^{(3 \mathrm{D})}(r, z)=0 .
$$

\section{Radial Component $H_{r}^{\text {(durand) }}(r, z)$}

We present here the expression of the radial component established by Durand [21] and consider it as $H_{r}^{(3 \mathrm{D})}(r, z)$, in so far as it is by now the simplest expression. According to Durand, the radial component $H_{r}^{\text {(durand) }}(r, z)$ is expressed as follows:

$$
\begin{aligned}
& H_{r}^{(\text {durand })}(r, z)=\frac{\sigma^{*}}{2 \pi \mu_{0}} \frac{\sqrt{\left(r_{\text {out }}+r\right)^{2}+(z-h)^{2}}}{r} \\
& \quad \times\left(\left(1-\frac{k_{1}^{2}}{2}\right) \mathbf{K}^{*}\left[k_{1}\right]-\mathbf{E}^{*}\left[k_{1}\right]\right) \\
& \quad-\frac{\sigma^{*}}{2 \pi \mu_{0}} \frac{\sqrt{\left(r_{\text {in }}+r\right)^{2}+(z-h)^{2}}}{r} \\
& \quad \times\left(\left(1-\frac{k_{2}^{2}}{2}\right) \mathbf{K}^{*}\left[k_{2}\right]-\mathbf{E}^{*}\left[k_{2}\right]\right)
\end{aligned}
$$

with

and

$$
\begin{aligned}
& \mathbf{K}^{*}[k]=\mathbf{K}^{*}\left[\frac{\pi}{2}, k\right]=\int_{0}^{\phi=\frac{\pi}{2}} \frac{d \theta}{\sqrt{1-k^{2} \sin (\theta)^{2}}} \\
& \mathbf{E}^{*}[k]=\mathbf{E}^{*}\left[\frac{\pi}{2}, k\right]=\int_{0}^{\phi=\frac{\pi}{2}} \sqrt{1-k^{2} \sin (\theta)^{2}} d \theta
\end{aligned}
$$

$$
\begin{aligned}
& k_{1}=\frac{2 \sqrt{r_{\text {out }} r}}{\sqrt{\left(r_{\text {out }}+r\right)^{2}+(z-h)^{2}}} \\
& k_{2}=\frac{2 \sqrt{r_{\text {in }} r}}{\sqrt{\left(r_{\text {in }}+r\right)^{2}+(z-h)^{2}}} .
\end{aligned}
$$

Fig. 2 represents the radial component $H r^{(\text {durand })}(r, z)$ versus the radial distance. 
TABLE I

DEFINITION OF THE PARAMETERS USED IN $(9)$
\begin{tabular}{|l|c|}
\hline Parameters & \\
\hline$a$ & $2(z-h)\left(r^{2}+(z-h)^{2}\right)$ \\
\hline$b_{1}$ & $2(z-h) r r_{o u t}$ \\
\hline$c_{1}$ & $r^{2}+r_{o u t}^{2}+(z-h)^{2}$ \\
\hline$d_{1}-2 r r_{\text {out }}$ \\
\hline$e$ & $-r^{2}-2(z-h)^{2}$ \\
\hline$f$ & $r^{2}$ \\
\hline$b_{2}$ & $2(z-h) r r_{\text {in }}$ \\
\hline$c_{2}$ & $r^{2}+r_{\text {in }}^{2}+(z-h)^{2}$ \\
\hline$d_{2}$ & $-2 r r_{\text {in }}$ \\
\hline
\end{tabular}

\section{E. Axial Component $H_{z}(r, z)$}

The axial component $H_{z}(r, z)$ is expressed as follows:

$$
H_{z}^{(3 \mathrm{D})}(r, z)=\frac{\sigma^{*}}{4 \pi \mu_{0}}\left(\gamma\left(\theta_{2}\right)-\gamma\left(\theta_{1}\right)\right)
$$

with

$$
\begin{aligned}
\gamma(\theta)= & h_{1}(\theta) \eta_{1}(\theta) \boldsymbol{\Pi}^{*}\left[\frac{2\left(c_{1}+d_{1}\right) f}{2 c_{1} f-\sqrt{2} \sqrt{d_{1}^{2} f(-e+f)}},\right. \\
& \left.i \sinh ^{-1}\left[\sqrt{\frac{-1}{c_{1}+d_{1}}} \sqrt{c_{1}-d_{1} \cos (\theta)}\right], \frac{c_{1}+d_{1}}{c_{1}-d_{1}}\right] \\
& +h_{2}(\theta) \eta_{1}(\theta) \boldsymbol{\Pi}^{*}\left[\frac{2\left(c_{1}+d_{1}\right) f}{2 c_{1} f+\sqrt{2} \sqrt{d_{1}^{2} f(-e+f)}},\right. \\
& \left.i \sinh ^{-1}\left[\sqrt{\frac{-1}{c_{1}+d_{1}}} \sqrt{c_{1}-d_{1} \cos (\theta)}\right], \frac{c_{1}+d_{1}}{c_{1}-d_{1}}\right] \\
& -h_{3}(\theta) \eta_{2}(\theta) \boldsymbol{\Pi}^{*}\left[\frac{2\left(c_{2}+d_{2}\right) f}{2 c_{2} f-\sqrt{2} \sqrt{d_{2}^{2} f(-e+f)}},\right. \\
& \left.i \sinh ^{-1}\left[\sqrt{\frac{-1}{c_{2}+d_{2}}} \sqrt{c_{2}-d_{2} \cos (\theta)}\right], \frac{c_{2}+d_{2}}{c_{2}-d_{2}}\right] \\
& -h_{4}(\theta) \eta_{2}(\theta) \boldsymbol{\Pi}^{*}\left[\frac{2\left(c_{2}+d_{2}\right) f}{2 c_{2} f+\sqrt{2} \sqrt{d_{2}^{2} f(-e+f)}},\right. \\
& \left.i \sinh ^{-1}\left[\sqrt{\frac{-1}{c_{2}+d_{2}}} \sqrt{c_{2}-d_{2} \cos (\theta)}\right], \frac{c_{2}+d_{2}}{c_{2}-d_{2}}\right](10)
\end{aligned}
$$

where $\Pi^{*}[n, \phi, m]$ is given in terms of the incomplete elliptic integral of the third kind by (11). Equation (10) contains the imaginary number $i\left(i^{2}=-1\right)$. However, the result $H_{z}^{(3 \mathrm{D})}(r, z)$ is a real number; we have not succeeded in obtaining a real expression for the axial component $H_{z}^{(3 \mathrm{D})}(r, z)$. The value $\theta_{2}$ equals 3.14, and the value $\theta_{1}$ equals -3.14 . If we take $\left|\theta_{2}\right|=$ $\left|\theta_{1}\right|=\pi,(10)$ is not definite. The closer the absolute value $\left|\theta_{1}\right|$ or $\left|\theta_{2}\right|$ is to $\pi$, the more precise the analytical expression is. The parameters used in (10) are defined in Table I. Moreover, when we input the expression (10) in Mathematica, we have to take the real part of $H z^{(3 \mathrm{D})}(r, z)$. Indeed, the imaginary part is the consequence of numerical noise and nearly equals zero

$$
\boldsymbol{\Pi}^{*}[n, \phi, m]=\int_{0}^{\phi} \frac{1}{\left(1-n \sin (\theta)^{2}\right) \sqrt{1-m \sin (\theta)^{2}}} d \theta
$$

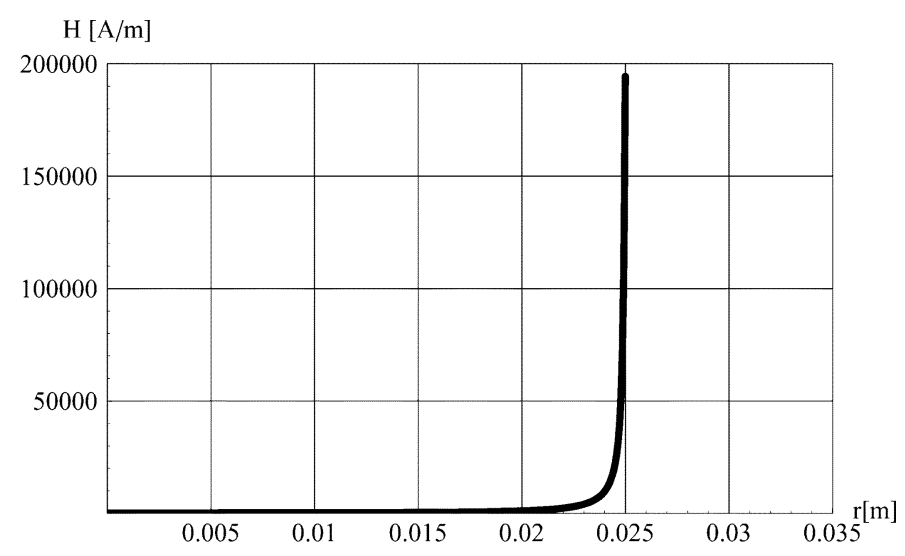

Fig. 3. The axial component $H_{z}(r, z)$ is a function of the radial length, the observation height is $z=4 \mathrm{~mm}, r_{\text {in }}=25 \mathrm{~mm}, r_{\text {out }}=28 \mathrm{~mm}, \sigma^{*}=1 \mathrm{~T}$.

$$
\begin{aligned}
\eta_{1}(\theta) & =\frac{\left(-i \sqrt{\frac{d_{1}(-1+\cos (\theta))}{c_{1}-d_{1}}} \sqrt{\frac{d_{1}(1+\cos (\theta))}{c_{1}+d_{1}}} \frac{1}{\cos (\theta)}\right)}{\left(2 \sqrt{\frac{-1}{c_{1}+d_{1}}} \sqrt{d_{1}^{2} f\left(-e+d_{1}\right)}\left(d_{1}^{2}(e-f)\right)+2 c_{1}^{2} f\right)} \\
\eta_{2}(\theta) & =\frac{\left(-i \sqrt{\frac{d_{2}(-1+\cos (\theta))}{c_{2}-d_{2}}} \sqrt{\frac{d_{2}(1+\cos (\theta))}{c_{2}+d_{2}}} \frac{1}{\cos (\theta)}\right)}{\left(2 \sqrt{\frac{-1}{c_{2}+d_{2}}} \sqrt{d_{2}^{2} f\left(-e+d_{2}\right)}\left(d_{2}^{2}(e-f)\right)+2 c_{2}^{2} f\right)} \\
h_{1}(\theta) & =2 a d_{1}\left(\sqrt{2} c_{1} f+\sqrt{d_{1}^{2} f(-e+f)}\right) \\
+ & b_{1}\left(\sqrt{2} d_{1}^{2}(e-f)-2 c_{1} \sqrt{d_{1}^{2} f(-e+f)}\right) \\
h_{2}(\theta) & =2 a d_{1}\left(-\sqrt{2} c_{1} f+\sqrt{d_{1}^{2} f(-e+f)}\right) \\
+ & b_{1}\left(\sqrt{2} d_{1}^{2}(-e+f)-2 c_{1} \sqrt{d_{1}^{2} f(-e+f)}\right) \\
h_{3}(\theta) & =2 a d_{2}\left(\sqrt{2} c_{2} f+\sqrt{d_{2}^{2} f(-e+f)}\right) \\
+ & b_{2}\left(\sqrt{2} d_{2}^{2}(e-f)-2 c_{2} \sqrt{d_{2}^{2} f(-e+f)}\right) \\
+ & b_{2}\left(\sqrt{2} d_{2}^{2}(-e+f)-2 c_{2} \sqrt{d_{2}^{2} f(-e+f)}\right) \\
h_{4}(\theta) & =2 a d_{2}\left(-\sqrt{2} c_{2} f+\sqrt{d_{2}^{2} f(-e+f)}\right) \\
&
\end{aligned}
$$

Fig. 3 shows the axial field component $H_{z}^{(3 \mathrm{D})}(r, z)$ as a function of the radial distance of the observation point $M(r, z)$ of the space. The ring inner radius $r_{\text {in }}$ equals $25 \mathrm{~mm}$, the ring outer one $r_{\text {out }}$ equals $28 \mathrm{~mm}$, and the ring height $h$ equals $3 \mathrm{~mm}$. The magnetic polarization $\boldsymbol{\sigma}^{*}$ is $1 \mathrm{~T}$. The axial field component $H_{z}^{(3 \mathrm{D})}(r, z)$ is plotted from $r=0 \mathrm{~mm}$ to $r=r_{\mathrm{in}}$. From a radial observation point higher than $r_{\text {in }}$, the axial field component $H_{z}^{(3 \mathrm{D})}(r, z)$ is more difficult to plot with Mathematica. 


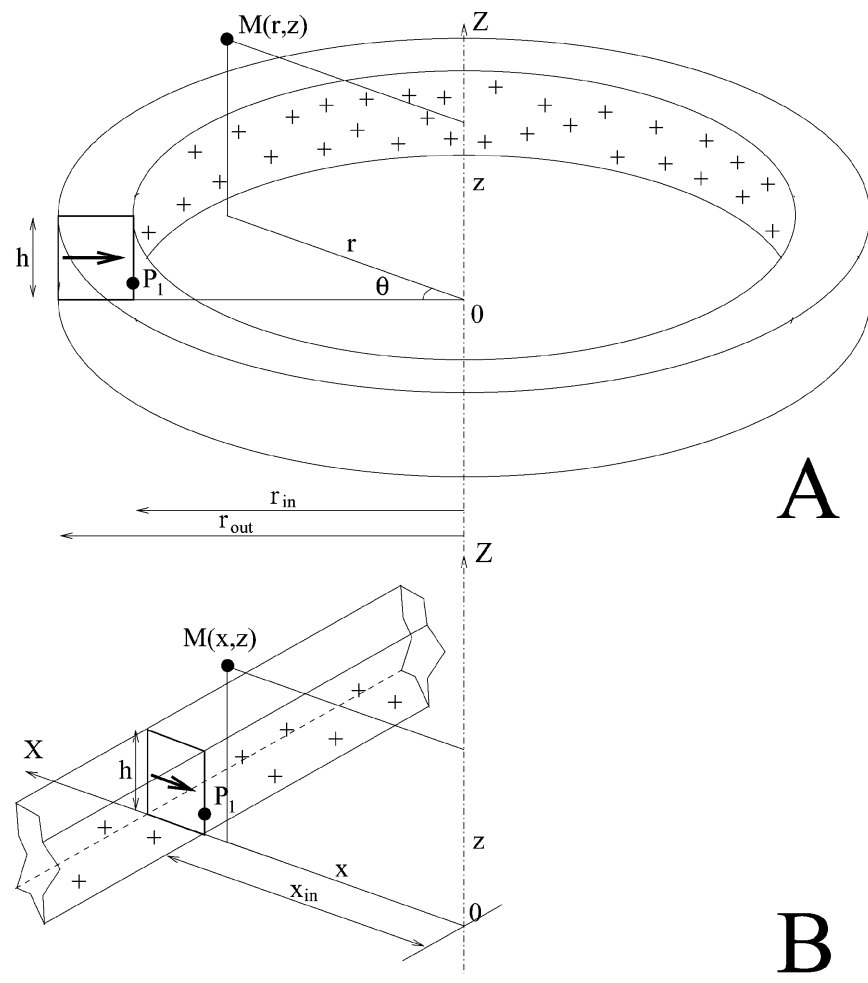

Fig. 4. Used geometries; A is a ring whose symmetry axis is $z$, its inner radius is $r_{\text {in }}$, its outer radius is $r_{\text {out }}$, its height is $h$, its magnetic polarization is radial: $\mathrm{B}$ is an infinitely long parallepiped, its height is $h, x_{\mathrm{in}}$ equals $r_{\mathrm{in}}$.

\section{Analytical Calculation of the Magnetic Field CREATED By RAdially Magnetized PERMANENT-MAGNET RINGS}

\section{A. Notation and Geometry}

The geometry and the related parameters are shown in Fig. 4(A). The axis $z$ is an axis of symmetry. Calculations are obtained by the use of the coulombian model. The permanent-magnet ring is thus represented by two curved planes which correspond here to the inner and outer faces of the ring. The inner one is charged with a surface magnetic pole density $+\boldsymbol{\sigma}^{*}$; the outer one is charged with the opposite surface magnetic pole density $-\boldsymbol{\sigma}^{*}$.

We only consider the ring inner face to simplify the analytical calculation.

Let us consider a point $P_{1}$ on the ring inner face. The magnetic field $\vec{H}$ created by the ring inner face at any point $M(r, z)$ of the space is given by

$$
\begin{aligned}
& \vec{H}=\frac{\sigma^{*}}{4 \pi \mu_{0}} \int_{\theta=0}^{\theta=2 \pi} \int_{z_{1}=0}^{z_{1}=h} \frac{\overrightarrow{P_{1} M}}{\left|\overrightarrow{P_{1} M}\right|^{3}} r_{1} d \theta d z_{1} \\
& \vec{H}(r, z)=\frac{\sigma^{*}}{4 \pi \mu_{0}} \int_{\theta=0}^{\theta=2 \pi} \int_{z_{1}=0}^{z_{1}=h} \\
& \times \frac{\left(r-r_{1} \cos (\theta)\right) \vec{u}_{r}-r_{1} \sin (\theta) \vec{u}_{\theta}+\left(z-z_{1}\right) \vec{u}_{z}}{\left.\left(r^{2}+r_{1}^{2}-2 r r_{1} \cos (\theta)+\left(z-z_{1}\right)^{2}\right)\right)^{\frac{3}{2}}} r_{1} d z_{1} d \theta .
\end{aligned}
$$

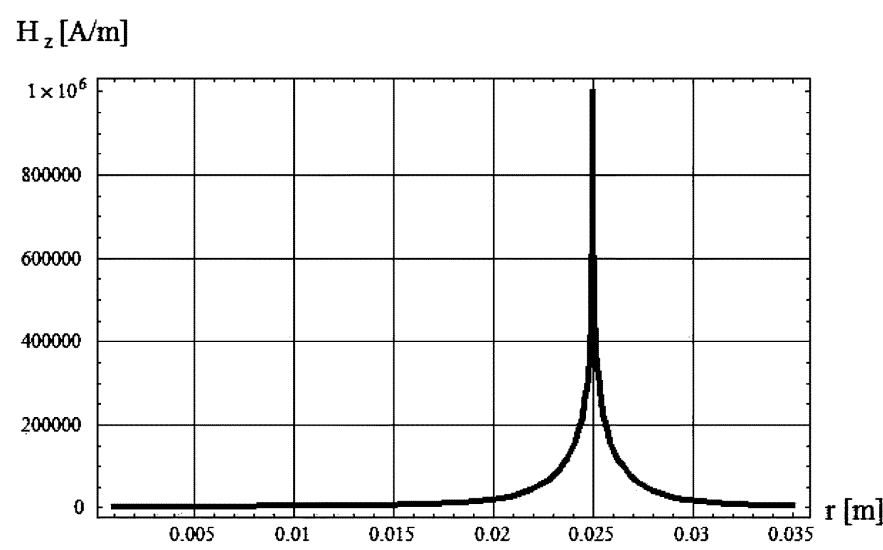

Fig. 5. Field axial component $H_{z}^{(3 \mathrm{D})}(r, z)$ versus the radial distance $r$ of the observation point. The observation height $z$ is $3 \mathrm{~mm}, r_{\text {in }}=25 \mathrm{~mm}, r_{\text {out }}=$ $28 \mathrm{~mm}, \sigma^{*}=1 \mathrm{~T}$.

\section{B. Components Along the Three Directions $\vec{u}_{r}, \vec{u}_{\theta}, \vec{u}_{z}$}

The integration of (18) leads to the magnetic field components $H_{r}^{(3 \mathrm{D})}(r, z), H_{\theta}^{(3 \mathrm{D})}(r, z), H_{z}^{(3 \mathrm{D})}(r, z)$ along the three defined axes (20), (21), (24).

\section{Azimuthal Component $H_{\theta}^{(3 \mathrm{D})}(r, z)$}

The azimuthal component $H_{\theta}^{(3 \mathrm{D})}(r, z)$ equals zero on account of the cylindrical symmetry:

$$
H_{\theta}^{(3 \mathrm{D})}(r, z)=0
$$

\section{Axial Component $H_{z}^{(3 \mathrm{D})}(r, z)$}

The axial component $H_{z}^{(3 \mathrm{D})}(r, z)$ is expressed as follows:

$$
\begin{aligned}
H_{z}^{(3 \mathrm{D})}(r, z) & =\frac{\sigma^{*}}{4 \pi \mu_{0}}\left(-4 r_{\mathrm{in}} \frac{\mathbf{K}^{*}\left[-\frac{-4 r r_{\mathrm{in}}}{r^{2}+r_{\mathrm{in}}^{2}-2 r r_{\mathrm{in}}+z^{2}}\right]}{\sqrt{r^{2}+r_{\mathrm{in}}^{2}-2 r r_{\mathrm{in}}+z^{2}}}\right. \\
+4 r_{\mathrm{in}} & \left.\frac{\mathbf{K}^{*}\left[-\frac{-4 r r_{\mathrm{in}}}{r^{2}+r_{\mathrm{in}}^{2}-2 r r_{\mathrm{in}}+(z-h)^{2}}\right]}{\sqrt{r^{2}+r_{\mathrm{in}}^{2}-2 r r_{\mathrm{in}}+(z-h)^{2}}}\right)
\end{aligned}
$$

where $\mathbf{K}^{*}[m]$ is given in terms of the incomplete elliptic integral of the first kind by

$$
\mathbf{K}^{*}[m]=\mathbf{F}^{*}\left[\frac{\pi}{2} \mid m\right]
$$

and $\mathbf{F}^{*}[\phi, m]$ is given in terms of the elliptic integral of the first kind by

$$
\mathbf{F}^{*}[\phi \mid m]=\int_{\theta=0}^{\theta=\phi} \frac{1}{\sqrt{1-m \sin (\theta)^{2}}} d \theta
$$

Fig. 5 shows the axial component $H_{z}^{(3 \mathrm{D})}(r, z)$ as a function of the radial distance of the observation point $M(r, z)$ for a given altitude $z=3 \mathrm{~mm}$. We observe a radial component peak which corresponds to the ring inner radius. 
TABLE II

DEFINITION OF THE PARAMETERS USED IN (24)

\begin{tabular}{|l|c|}
\hline Parameters & \\
\hline$a_{1}$ & $r_{i n} r z$ \\
\hline$b_{1}$ & $-r_{i n}^{2} z$ \\
\hline$c$ & $r^{2}+r_{i n}^{2}$ \\
\hline$d$ & $-2 r r_{i n}$ \\
\hline$e_{1}$ & $z^{2}$ \\
\hline$a_{2}$ & $-r_{i n} r(z-h)$ \\
\hline$b_{2}$ & $r_{i n}^{2}(z-h)$ \\
\hline$e_{2}$ & $(z-h)^{2}$ \\
\hline
\end{tabular}

\section{E. Radial Component $H_{r}^{(3 \mathrm{D})}(r, z)$}

The radial component of the field $H_{r}^{(3 \mathrm{D})}(r, z)$ is expressed as follows:

$$
H_{r}^{(3 \mathrm{D})}(r, z)=\frac{\sigma^{*}}{2 \pi \mu_{0}}\left(\beta\left(u_{1}\right)-\beta\left(u_{2}\right)\right)
$$

with (25), shown at the bottom of the page, where $F^{*}[\phi, m]$ is defined by (23) and $\Pi^{*}[n, \phi, m]$ is given by (11). Equation (25) contains the imaginary number $i\left(i^{2}=-1\right)$. However, the result $H_{r}^{(3 \mathrm{D})}(r, z)$ is a real number; we have not succeeded in obtaining a real expression for the radial component $H_{r}^{(3 \mathrm{D})}(r, z)$. The value $u_{2}$ equals 0.999999999 and the value $u_{1}$ equals -0.999999999 . If we take $\left|u_{2}\right|=\left|u_{1}\right|=1$, the expression (25) is not definite. The closer the absolute value $\left|u_{1}\right|$ or $\left|u_{2}\right|$ is to one, the more precise the analytical expression is. The parameters used in (25) are defined in Table II. Moreover, when we input the expression (25) in Mathematica, we have to take the real part of $H r^{(3 \mathrm{D})}(r, z)$. The imaginary part corresponds to numerical noise and nearly equals zero.

Fig. 6 shows the radial field component $H_{r}^{(3 \mathrm{D})}(r, z)$ as a function of the radial distance of the observation point $M(r, z)$ of the space. The ring inner radius $r_{\text {in }}$ is $25 \mathrm{~mm}$, the outer one $r_{\text {out }}$ is $28 \mathrm{~mm}$, and the ring height $h$ is $3 \mathrm{~mm}$. The magnetic polarization $\sigma^{*}$ equals $1 \mathrm{~T}$. We observe a discontinuity of the radial field component for a distance which corresponds to the ring inner

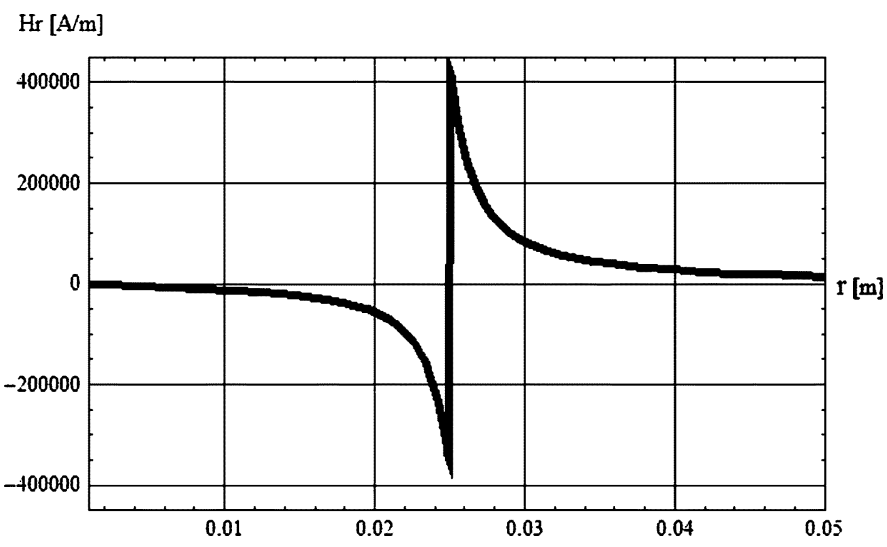

Fig. 6. Radial field component $H r^{(3 \mathrm{D})}(r, z)$ as a function of the radial distance $r$ of the observation point $M(r, z)$. The observation height $z$ is $1,5 \mathrm{~mm}$, $r_{\text {in }}=25 \mathrm{~mm}, r_{\text {out }}=28 \mathrm{~mm}, h=3 \mathrm{~mm}, \sigma^{*}=1 T$.

radius $r_{\text {in }}$. The ring radial component $H r^{(3 \mathrm{D})}(r, z)$ verifies the Gauss theorem (26), which is consistent with the expressions obtained

$$
\int_{\theta=0}^{\theta=2 \pi} \int_{z=0}^{z=h} \vec{H} \cdot d \vec{S}=\frac{\sigma^{*}}{4 \pi \mu_{0}} .
$$

\section{COMPARISON BetweEn the APPROXimate 2-D ANALYTICAL APPROACH AND THE EXACT 3-D ANALYTICAL APPROACH}

This section discusses the utility of a 3-D analytical approach to calculate the magnetic field created by radially magnetized permanent-magnet rings. For this purpose, we calculate the relative difference between the magnetic field components calculated with the approximate 2-D analytical approach and those calculated with the exact 3-D analytical approach.

\section{A. The 2-D Analytical Approach}

Fig. 4(B) shows the two geometries considered in the comparison of the 2-D and the 3-D analytical approaches. The geometry

$$
\begin{aligned}
\beta(u)= & \left(\frac{2 i(1+u) \sqrt{\frac{d(-1+u)}{c+e_{1}+d u}}\left(-\left(a_{1} d+b_{1}\left(c+e_{1}\right)\right)\right) \mathbf{F}^{*}\left[i \sinh ^{-1}\left[\frac{\sqrt{-c+d-e_{1}}}{\sqrt{c+e_{1}+d u}}\right], \frac{c+d+e_{1}}{c-d+e_{1}}\right]}{d \sqrt{-c+d-e_{1}} e_{1} \sqrt{\frac{d(1+u)}{c+e_{1}+d u}} \sqrt{1-u^{2}}}\right) \\
& +\left(\frac{2 i(1+u) \sqrt{\frac{d(-1+u)}{c+e_{1}+d u}}\left(b_{1} c-a_{1} d\right) \mathbf{\Pi}^{*}\left[\frac{e_{1}}{c-d+e_{1}}, i \sinh ^{-1}\left[\frac{\sqrt{-c+d+e_{1}}}{c+e_{1}+d u}\right], \frac{c+d+e_{1}}{c-d+e_{1}}\right]}{d \sqrt{-c+d-e_{1}} e_{1} \sqrt{\frac{d(1+u)}{c+e_{1}+d u}} \sqrt{1-u^{2}}}\right) \\
& +\left(\frac{2 i(1+u) \sqrt{\frac{d(-1+u)}{c+e_{2}+d u}}\left(-\left(a_{2} d+b_{2}\left(c+e_{2}\right)\right)\right) \mathbf{F}^{*}\left[i \sinh ^{-1}\left[\frac{\sqrt{-c+d-e_{2}}}{\sqrt{c+e_{2}+d u}}\right], \frac{c+d+e_{2}}{c-d+e_{2}}\right]}{d \sqrt{-c+d-e_{2}} e_{2} \sqrt{\frac{d(1+u)}{c+e_{2}+d u}} \sqrt{1-u^{2}}}\right) \\
& +\left(\frac{2 i(1+u) \sqrt{\frac{d(-1+u)}{c+e_{2}+d u}}\left(b_{2} c-a_{2} d\right) \mathbf{\Pi}^{*}\left[\frac{e_{2}}{c-d+e_{2}}, i \sinh ^{-1}\left[\frac{\sqrt{-c+d+e_{2}}}{c+e_{2}+d u}\right], \frac{c+d+e_{2}}{c-d+e_{2}}\right]}{d \sqrt{-c+d-e_{2}} e_{2} \sqrt{\frac{d(1+u)}{c+e_{2}+d u}} \sqrt{1-u^{2}}}\right)
\end{aligned}
$$


which corresponds to the 2-D analytical approach is an infinite parallelepiped whose height is $h$. This parallelepiped can be represented by two parallel planes. The inner one is charged with a surface magnetic pole density $+\sigma^{*}$; the outer one is charged with the opposite surface magnetic pole density $-\sigma^{*}$. Let us consider a point on the plane. We only calculate the magnetic field created by the face $+\sigma^{*}$. The magnetic field $\vec{H}$, at any point $M(x, y, z)$ of the space, is given by

$$
\vec{H}=\frac{\sigma^{*}}{4 \pi \mu_{0}} \int_{y_{1}=-\infty}^{y_{1}=+\infty} \int_{z_{1}=0}^{z_{1}=b} \frac{\overrightarrow{P_{1} M}}{\left|\overrightarrow{P_{1} M}\right|^{3}} d y_{1} d z_{1} .
$$

The integration of (27) leads to the magnetic field components $H_{r}^{(2 \mathrm{D})}(r, z)$ and $H_{z}^{(3-D)}(r, z)$ along the defined axes, which are given by the expressions of (28) and (29)

$H_{r}^{(2-D)}(r, z)=\frac{\sigma^{*}}{2 \pi \mu_{0}}\left(\arctan \left[\frac{z}{r-r_{1}}\right]-\arctan \left[\frac{z-h}{r-r_{1}}\right]\right)$

$H_{z}^{(2-D)}(r, z)=\frac{\sigma^{*}}{4 \pi \mu_{0}} \log \left[\frac{\left(\left(r-r_{\text {in }}\right)^{2}+z^{2}\right)}{\left(\left(r-r_{\text {in }}\right)^{2}+(z-h)^{2}\right)}\right]$.

These expressions were established by J. P. Yonnet [12].

\section{B. Comparison Between the 2-D Analytical Approach and the 3-D Analytical Approach for a Given Ring Inner Radius}

The aim of this section is to show the limits of the 2-D analytical approach.

1) Radial Component $H_{r}(r, z)$ : Let us consider the radial component $H_{r}(r, z)$. We calculate the relative difference $\left(\Delta H_{r} / H_{r}\right)$ with the expressions of (24) and (28). The relative difference $\left(\Delta H_{r} / H_{r}\right)$ is defined in

$$
\frac{\Delta H_{r}}{H_{r}}=\left|\frac{H_{r}^{(3 \mathrm{D})}(r, z)-H_{r}^{(2 \mathrm{D})}(r, z)}{H_{r}^{(3 \mathrm{D})}(r, z)}\right| .
$$

We plot $\left(\Delta H_{r} / H_{r}\right)$ in Fig. 7. This figure shows that the 2-D analytical approach is not sufficient to calculate the radial component $H_{r}(r, z)$ either close to or far from the permanent-magnet ring. We incur an error of at least $10 \%$ in calculating the radial component by using the 2-D analytical approach.

2) Axial Component $H_{z}(r, z)$ : Let us consider the axial component $H_{z}(r, z)$. We calculate the relative difference $\left(\Delta H_{z} / H_{z}\right)$ by using the expressions of (21) and (29). The relative difference $\left(\Delta H_{z} / H_{z}\right)$ is defined by

$$
\frac{\Delta H_{z}}{H_{z}}=\left|\frac{H_{z}^{(3 \mathrm{D})}(r, z)-H_{z}^{(2 \mathrm{D})}(r, z)}{H_{z}^{(3 \mathrm{D})}(r, z)}\right| .
$$

The relative difference $\left(\Delta H_{z} / H_{z}\right)$ is plotted in Fig. 8. This figure shows that for a given inner radius which equals $25 \mathrm{~mm}$, we can use the 2-D analytical approach with a $2 \%$ relative error to calculate the axial component $H_{z}(r, z)$ at a radial distance from the ring smaller than $1 \mathrm{~mm}$. We deduce that the 2-D analytical approach is a good approximation for calculating the

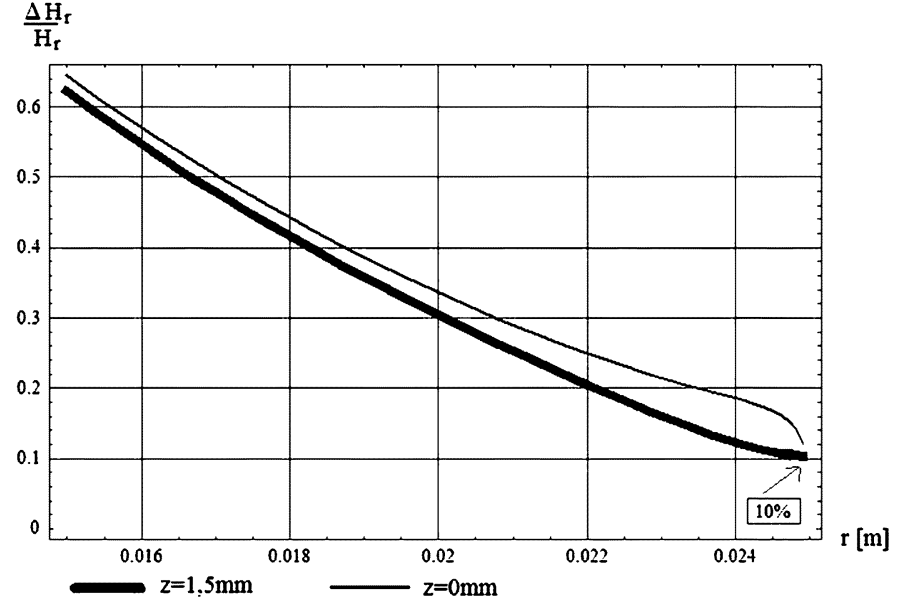

Fig. 7. Representation of the relative difference between the radial component calculated with the 2-D analytical approach and the one calculated with the 3-D analytical approach; the relative difference $\left(\Delta H_{r} / H_{r}\right)$ is a function of the radial length $r ; r_{\text {in }}=x_{\text {in }}=25 \mathrm{~mm}, r_{\text {out }}=x_{\text {out }}=28 \mathrm{~mm}, h=3 \mathrm{~mm}$, $\sigma^{*}=1 \mathrm{~T}$.

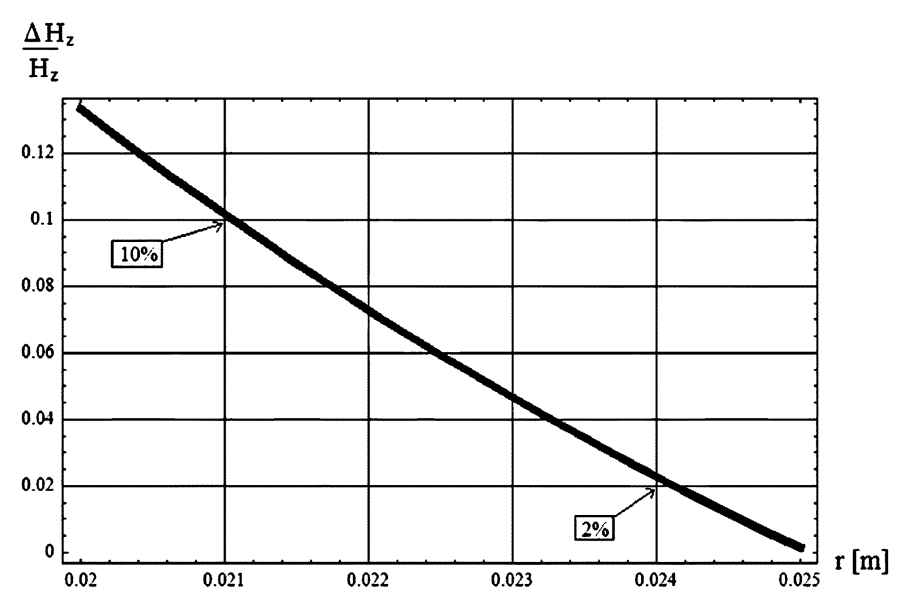

Fig. 8. Representation of the relative difference between the axial component calculated by the 2-D analytical approach and the 3-D analytical approach; the relative difference $\left(\Delta H_{z} / H_{z}\right)$ is a function of the radial distance $r$; the observation height is $z=0 \mathrm{~mm} ; r_{\text {in }}=x_{\text {in }}=25 \mathrm{~mm}, r_{\text {out }}=x_{\text {out }}=28 \mathrm{~mm}$, $h=3 \mathrm{~mm}, \sigma^{*}=1 \mathrm{~T}$.

magnetic field near the permanent-magnet ring. The discrepancies between the 2-D and the 3-D approaches are far larger for the radial field component than for the axial one. In conclusion, Figs. 7 and 8 show that the 2-D analytical approach is only appropriate for calculating the axial field component $H_{z}(r, z)$ for a inner radius which equals $25 \mathrm{~mm}$. However, the 2-D analytical approach is a good approximation for calculating the radial field component $H_{r}(r, z)$ in this case.

\section{Radius of Curvature Influence on the Magnetic Field}

In this section, we study the radius of curvature influence on the magnetic field created by the permanent-magnet ring. For this purpose, we calculate the magnetic field components $H_{r}$ and $H_{z}$ at a given observation point for different radii of curvature. 


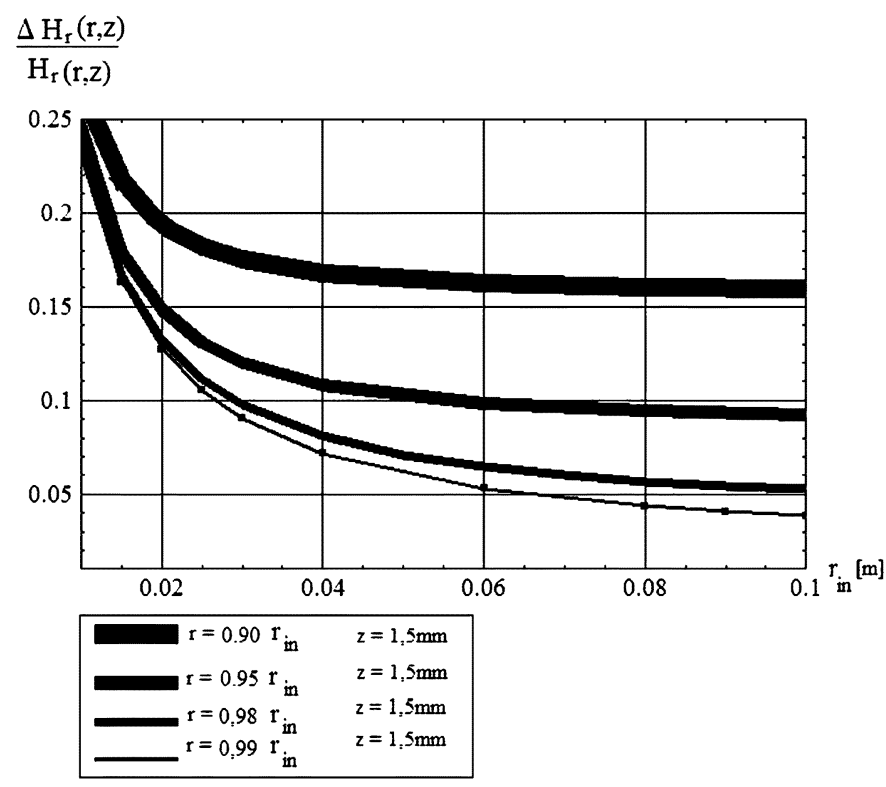

Fig. 9. The relative difference $\left(\Delta H_{r} / H_{r}\right)$ is a function of the radius of curvature $r$; the observation height is $z=1,5 \mathrm{~mm} ; r_{\text {in }}=x_{\text {in }}=25 \mathrm{~mm}$, $r_{\text {out }}=x_{\text {out }}=28 \mathrm{~mm}, h=3 \mathrm{~mm}, \sigma^{*}=1 \mathrm{~T}$.

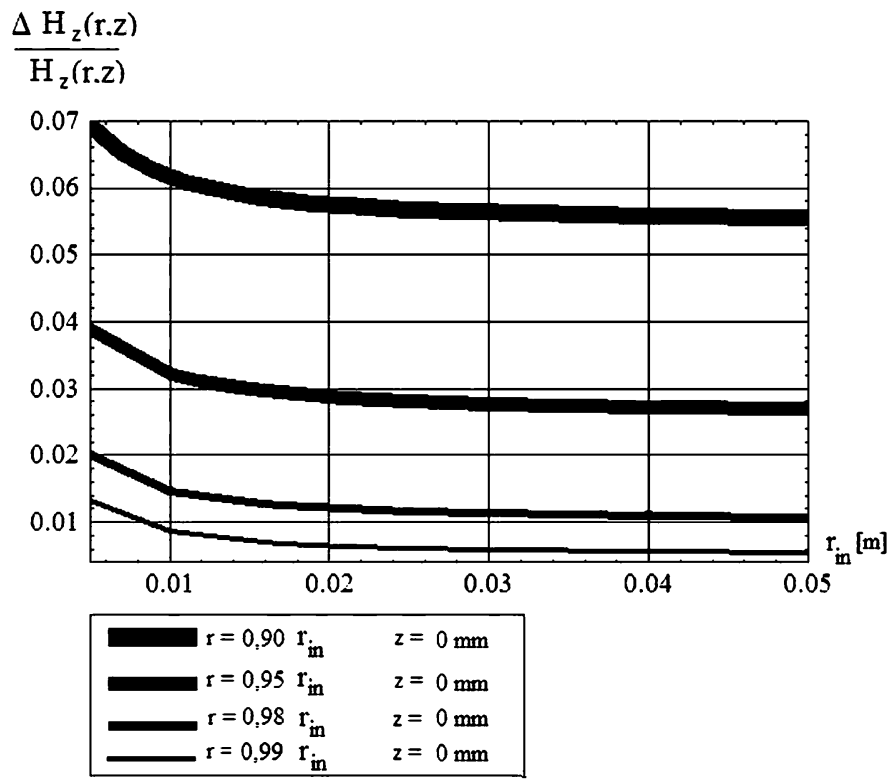

Fig. 10. The relative difference $\left(\Delta H_{z} / H_{z}\right)$ is a function of the radius of curvature $r ; r_{\text {in }}=x_{\text {in }}=25 \mathrm{~mm}, r_{\text {out }}=x_{\text {out }}=28 \mathrm{~mm}, h=3 \mathrm{~mm}, \sigma^{*}=1 \mathrm{~T}$.

\section{Radius of Curvature Influence on $H_{r}(r, z)$}

In Fig. 9, the relative difference $\left(\Delta H_{r} / H_{r}\right)$ (30) is a function of the radius of curvature. The relative difference $\left(\Delta H_{r} / H_{r}\right)$ is plotted for different radii of curvature $r_{\text {in }}$ in Fig. 9. This figure shows that the closer the observation point is to the radius of curvature, the more precise the 2-D analytical approach is. However, when the radius of curvature is smaller than $60 \mathrm{~mm}$, the 2-D analytical approach is not appropriate since the relative error is at least $5 \%$. In consequence, we should use the 3-D analytical approach to calculate the radial component of the field created by a permanent-magnet ring.

\section{E. Radius of Curvature Influence on $H_{z}(r, z)$}

In Fig. 10, the relative difference $\left(\Delta H_{z} / H_{z}\right)$ is a function of the radius of curvature.

The relative difference $\left(\Delta H_{z} / H_{z}\right)$ becomes important for a ring radius of curvature which equals $10 \mathrm{~mm}$. This figure shows that the 2-D analytical approach is only appropriate when the observation point distance $r$ is smaller than $0,95 r_{c}$. When $r=0,95 r_{c}$, an at least 5\% error is incured when the 2-D analytical approach is used to calculate the axial component. However, for an observation point distance larger than $0,95 r_{c}$, the error is smaller than $4 \%$. In conclusion, the $3-\mathrm{D}$ analytical calculation is necessary to determine at any point of the space the radial component $H_{r}(r, z)$ and the axial component $H_{z}(r, z)$ but the 2-D analytical approach can be used to calculate the axial component $H_{z}(r, z)$ when we calculate the axial component for an observation point distance which equals at least $0,95 r_{c}$. The 2-D analytical approach cannot be used to calculate the radial component $H_{r}(r, z)$ if the radius of curvature is smaller than $60 \mathrm{~mm}$.

\section{CONCLUSION}

This paper presents the 3-D analytical calculation of the field intensity created by ring magnets. For axially magnetized ring magnets, the paper gives a formulation of both the radial component and the axial component. The formulation of the axial component has been established but this expression is difficult to plot in the whole space. It is to be noted that this paper models the magnet as a ring plane charged by a surface density.

For radially magnetized ring magnets, the paper models the magnet as a cylindrical plane charged by a surface density, and the axial and radial components of the field are given analytically. Furthermore, a comparison has been made between the approximate 2-D analytical formulation and the 3-D analytical formulation for radially polarized magnets. The result is that the radial component becomes rapidly inaccurate with the 2-D formulation when the distance of the observation point from the magnet increases and when the radius of the magnet is smaller than $60 \mathrm{~mm}$. Indeed, the relative difference between 2-D and 3 -D values remains under $5 \%$ if the magnet curvature is larger than $60 \mathrm{~mm}$ and if the observation point is very close to the magnet, at a distance smaller than $1 \%$ of the magnet radius. Hence, the calculation of the axial component can often require the 3-D formulation. The 2-D formulation has a larger range of validity for the axial component, as the relative difference between 2-D and 3-D values remains under $6 \%$ for magnet radii of $10 \mathrm{~mm}$ and an observation point distance from the magnet representing $10 \%$ of the magnet radius. The result of the comparison should help to decide whether the 2-D or the 3-D analytical formulation must be chosen to dimension and optimize a specific device.

The Mathematica files containing the analytical expressions used to calculate the axial and radial components for axial and radial magnetizations are given online [37].

\section{REFERENCES}

[1] M. Abele and H. A. Leupold, "A general method for flux confinement in permanent magnet structure," J. Appl. Phys., vol. 64, no. 10, pp. $5988-5990,1988$. 
[2] M. Abele, J. Jensen, and H. Rusinek, "Generation of uniform high fields with magnetized wedges," IEEE Trans. Magn., vol. 33, no. 5, pp. 3874-3876, Sep. 1997.

[3] K. Halbach, "Design of permanent multiple magnets with oriented rec material," Nucl. Inst. Meth., vol. 169, pp. 1-10, 1980.

[4] Y. Pulyer and M. Hrovat, "Generation of remote homogeneous magnetic fields," IEEE Trans. Magn., vol. 38, no. 3, pp. 1553-1563, May 2002.

[5] C. Blache and G. Lemarquand, "High magnetic field gradients in flux confining permanent magnet structures," J. Magn. Magn. Mater., vol. 104-107, pp. 1111-1112, 1992.

[6] O. Cuguat, J. Delamare, and G. Reyne, "Magnetic micro-actuators and systems (magmas)," IEEE Trans. Magn., vol. 39, no. 6, pp. 3607-3612, Nov. 2003.

[7] H. Chetouani, C. Jeandey, V. Haguet, H. Rostaing, C. Dieppedale, and G. Reyne, "Diamagnetic levitation with permanent magnets for contactless guiding and trapping microdroplets and particles in air and liquids," IEEE Trans. Magn., vol. 42, no. 10, pp. 3557-3559, Oct. 2006.

[8] J. Wang, G. W. Jewell, and D. Howe, "Design optimisation and comparison of permanent magnet machines topologies," IEE. Proc. Elect. Power Appl., vol. 148, pp. 456-464, Sep. 2001.

[9] G. Lemarquand and V. Lemarquand, "Annular magnet position sensor," IEEE. Trans. Magn., vol. 26, no. 5, pp. 2041-2043, Sep. 1990.

[10] G. Lemarquand and V. Lemarquand, "Variable magnetic torque sensor," J. Appl. Phys., vol. 70, no. 10, pp. 6630-6632, 1991.

[11] M. Marinescu and N. Marinescu, "Compensation of anisotropy effects in flux-confining permanent-magnet structures," IEEE Trans. Magn., vol. 25, no. 5, pp. 3899-3901, Sep. 1989.

[12] J. P. Yonnet, Rare-Earth Iron Permanent Magnets. Oxford, U.K.: Oxford Science, 1996, ch. Magnetomechanical devices.

[13] F. Bancel and G. Lemarquand, "Three-dimensional analytical optimization of permanent magnets alternated structure," IEEE Trans. Magn., vol. 34, no. 1, pp. 242-247, Jan. 1998.

[14] M. Berkouk, V. Lemarquand, and G. Lemarquand, "Analytical calculation of ironless loudspeaker motors," IEEE Trans. Magn., vol. 37, no. 2, pp. 1011-1014, Mar. 2001.

[15] G. Lemarquand, "Ironless loudspeakers," IEEE Trans. Magn., vol. 43, no. 8, pp. 3371-3374, Aug. 2007.

[16] G. Lemarquand and V. Lemarquand, "Calculation method of permanent magnet pickups for electric guitars," IEEE Trans. Magn., vol. 43, no. 9, pp. 3573-3578, Sep. 2007.

[17] J. P. Yonnet, "Permanent magnet bearings an couplings," IEEE Trans. Magn., vol. 17, no. 1, pp. 1169-1173, Jan. 1981.

[18] Z. Q. Zhu, G. W. Jewell, and D. Howe, "Design considerations for permanent magnet polarised electromagnetically actuated brakes," IEEE Trans. Magn., vol. 31, no. 6, pp. 3743-3745, Nov. 1995.

[19] V. Lemarquand, J. F. Charpentier, and G. Lemarquand, "Nonsinusoidal torque of permanent-magnet couplings," IEEE Trans. Magn., vol. 35, no. 5, pp. 4200-4205, Sep. 1999.

[20] H. C. Yu, T. Y. Lee, S. J. Wang, M. L. Lai, J. J. Ju, D. R. Huang, and S. K. Lin, "Design of a voice coil motor used in the focusing system of a digital video camera," IEEE Trans. Magn., vol. 41, no. 10, pp. 3979-3981, Oct. 2005.

[21] E. Durand, "Electrostatique," Masson Editeur, Paris, France, vol. 1, pp. 248-251, 1964.
[22] E. P. Furlani, "Field analysis and optimization of ndfeb axial field permanent magnet motors," IEEE Trans. Magn., vol. 33, no. 5, pp. 3883-3885, Sep. 1997.

[23] Y. Zhilichev, "Calculation of magnetic field of tubular permanent magnet assemblies in cylindrical bipolar coordinates," IEEE Trans. Magn., vol. 43, no. 7, pp. 3189-3195, Jul. 2007.

[24] E. P. Furlani, S. Reznik, and A. Kroll, "A three-dimensonal field solution for radially polarized cylinders," IEEE Trans. Magn., vol. 31, no. 1, pp. 844-851, Jan. 1995.

[25] H. Rakotoarison, J. Yonnet, and B. Delinchant, "Using coulombian approach for modelling scalar potential and magnetic field of a permanent magnet with radial polarization," IEEE Trans. Magn., vol. 43, no. 4, pp. 1261-1264, Apr. 2007.

[26] J. P. Selvaggi, S. Salon, O. M. Kwon, and M. V. Chari, "Calculating the external magnetic field from permanent magnets in permanent-magnet motors - an alternative method," IEEE Trans. Magn., vol. 40, no. 5, pp. 3278-3285, Sept. 2004.

[27] J. P. Selvaggi, S. Salon, O. M. Kwon, M. V. K. Chari, and M. DeBortoli, "Computation of the external magnetic field, near-field or far-field from a circular cylindrical magnetic source using toroidal functions," IEEE Trans. Magn., vol. 43, no. 4, pp. 1153-1156, Apr. 2007.

[28] B. Azzerboni and E. Cardelli, "Magnetic field evaluation for disk conductors," IEEE Trans. Magn., vol. 29, no. 6, pp. 2419-2421, Nov. 1993.

[29] B. Azzerboni, E. Cardelli, M. Raugi, A. Tellini, and G. Tina, "Magnetic field evaluation for thick annular conductors," IEEE Trans. Magn., vol. 29, no. 3, pp. 2090-2094, May 1993.

[30] B. Azzerboni, E. Cardelli, M. Raugi, A. Tellini, and G. Tina, "Analytic expressions for magnetic field from finite curved conductors," IEEE Trans. Magn., vol. 27, no. 2, pp. 750-757, Mar. 1991.

[31] B. Azzerboni and G. Saraceno, "Three-dimensional calculation of the magnetic field created by current-carrying massive disks," IEEE Trans. Magn., vol. 34, no. 5, pp. 2601-2604, Sept. 1998.

[32] B. Azzerboni, E. Cardelli, and A. Tellini, "Computation of the magnetic field in massive conductor systems," IEEE Trans. Magn., vol. 25, no. 6, pp. 4462-4473, Nov. 1989.

[33] B. Azzerboni, E. Cardelli, and A. Tellini, "Analysis of the magnetic field distribution in an homopolar generator as a pulse power source of electromagnetic launchers," IEEE Trans. Magn., vol. 24, no. 1, pp. 495-499, Jan. 1988.

[34] S. Babic, C. Akyel, S. Salon, and S. Kincic, "New expressions for calculating the magnetic field created by radial current in massive disks," IEEE Trans. Magn., vol. 38, no. 2, pp. 497-500, Mar. 2002.

[35] S. Babic and M. Gavrilovic, "New expression for calculating magnetic fields due to current-carrying solid conductors," IEEE Trans. Magn., vol. 33, no. 5, pp. 4134-4136, Sep. 1997.

[36] S. Babic et al., "Analytical magnetostatic field calculation for the conductor carrying constant current in the longitudinal diretion," J. Appl. Phys., vol. 67, pp. 5827-5829, May 1990.

[37] [Online]. Available: http://www.univ-lemans.fr/ glemar/mathfiles. htm

Manuscript received December 10, 2007; revised March 20, 2008. Corresponding author: G. Lemarquand (e-mail: guy.lemarquand@univ-lemans.fr). 